CARTA AL EDITOR

\title{
Inventario para los Trastornos Afectivos y la Esquizofrenia para el episodio presente en niños en edad escolar (K-SADS-P): un estudio piloto de la fiabilidad entre jueces para niños y adolescentes griegos
}

\author{
G. Kolaitis, T. Korpa, I. Kolvin, J. Tsiantis \\ Departamento de Psiquiatría Infantil, Facultad de Medicina de la Universidad de Atenas, Hospital Infantil \\ "Aghia Sophia", Thivon and Levathias Street, 11527 Athens, Grecia.
}

El Inventario Infantil para los Trastornos Afectivos y la Esquizofrenia (K-SADS) es uno de los ejemplos más populares en psiquiatría infantil de instrumento de entrevista semiestructurado $[1,2,4]$. Lo desarrollaron Puig-Antich y Chambers basado en el Inventario para los Trastornos Afectivos y la Esquizofrenia (SADS) diseñado para adultos [7]. Chambers, PuigAntich y cols. llevaron a cabo el primer estudio de fiabilidad de la K-SADS-P-II, pero esta primera versión de la K-SADS no era plenamente compatible con el DSM-III. Revisiones e investigaciones más recientes proporcionan datos de que Ia K-SADS es una medida fiable de la depresión, la ansiedad, el trastorno emocional y el disocial (conduct disorder) [8].

El propósito de este estudio piloto era examinar la fiabilidad entre jueces de la versión del estado actual de la K-SADS para niños y adolescentes griegos en una población derivada clínicamente. La K-SADS-P se tradujo al griego (entre otras lenguas europeas) y luego hacia atrás al inglés. Los participantes fueron 26 niños y adolescentes (7-15 años de edad, 15 chicos y 11 chicas) y sus padres, que fueron derivados a nuestro departamento y entrevistados por dos entrevistadores adiestrados. Se erigieron escalas de grupos de síntomas de depresión, somatización, problemas de ansiedad y de conducta y se emprendió la fiabilidad entre jueces. Se llevaron a cabo entrevistas diagnósticas (separadamente con padre e hijo, que derivaron en una evaluación de resumen) basadas en los criterios del DSM-III-R [3] para el trastorno depresivo, el trastorno de ansiedad, el trastorno disocial y también codificaciones en la versión de la K-SADS-P de la Escala de Evaluación Global de Niños (CGAS) [6, 9, 10].
Las cuatro Escalas Resumen de Depresión de la KSADS-P, compuestas de 2-17 síntomas [4] mostraron una elevada fiabilidad entre jueces $(>0,96)$ y coherencia interna de moderada a alta $(>0,46)$. El estadístico Kappa [5] del diagnóstico para los trastornos depresivos fue 0,9. Se puso de manifiesto que cuanto mayor sea el número de elementos que representen este síndrome, más alto será el acuerdo (correlación) y esto está de acuerdo con lo esperado.

La fiabilidad entre jueces de la evaluación de los síntomas de ansiedad era más baja $(r=0,59)$ que para los trastornos depresivos y la coherencia interna era baja ( $\alpha$ de Cronbach $=0,44)$. La fiabilidad del diagnóstico de trastorno de ansiedad era más alta (Kappa $=0,8)$ que la comunicada por Chambers y cols. [4] $($ Kappa $=0,24)$.

La Escala de Somatización de la K-SADS-P (dolores e hipocondría) tuvo también una fiabilidad $(0,67)$ y consistencia interna $(0,49)$ más bajas, pero aceptables, que lo encontrado con los trastornos depresivos. La variable (escala) compuesta de conducta mostró fiabilidad alta $(0,83)$ así como consistencia interna elevada $(0,89)$. La kappa para los trastornos disociales fue también alta (Kappa $=0,9)$. La síntesis de los datos padre/hijo de la K-SADS-P proporcionó información que permitió codificaciones en la CGAS, que tuvo una fiabilidad entre jueces excelente (coeficiente de correlación intraclase: $0,91, P<0,001$ ).

En conclusión, los resultados de este estudio sobre las propiedades psicométricas de la K-SADS-P para la población psiquiátrica infantil y adolescente griega son prometedores. La limitación principal del presente estudio fue el tamaño relativamente pequeño de la muestra,

\footnotetext{
Kolaitis G, Korpa T, Kolvin I, Tsiantis J. Schedule for affective disorders and schizophrenia for school-age children-present episode (K-SADS-
} P): a pilot inter-rater reliability study for Greek children and adolescents. Eur Psychiatry 2003; 18: 374-375. 
adecuado sin embargo para los propósitos actuales. La segunda limitación es que se trata de una muestra clínica y, por tanto, los excelentes resultados de fiabilidad que se registran no son generalizables necesariamente a muestras comunitarias. Sin embargo, estas limitaciones no disminuyen la importancia de este estudio piloto sobre un instrumento diagnóstico muy útil para una población infantil psiquiátrica en Grecia y sus resultados prometedores para otros estudios similares como el que los mismos autores han emprendido sobre las propiedades psicométricas de una versión más reciente de la entrevista, la K-SADS-PL [8].

\section{AGRADECIMIENTOS}

A los autores les gustaría dar las gracias al Dr. Paul Ambrosini por sus útiles comentarios y sugerencias.

\section{BIBLIOGRAFÍA}

1 Ambrosini PJ, Metz C, Prabucki K, Lee JC. Videotape reliability of the third revised edition of the K-SADS. J Am Acad Child Adolesc Psychiatry 1989; 28: 723-8.

2 Ambrosini PJ. Historical development and present status of the schedule for affective disorders and schizophrenia for school-age children (K-SADS). J Am Acad Child Adolesc Psychiatry 2000; 39: 49-58.

3 American Psychiatric Association. Diagnostic and sta- tistical manual of mental disorders. 3rd ed. revised. Washington, DC: APA; 1987.

4 Chambers W, Puig-Antich J, Hirsch M, Paez P, Ambrosini $\mathrm{P}$, Tabrizi MA, et al. The assessment of affective disorders in children and adolescente by semistructured interview: test-retest reliability of the schedule for affective disorders and schizophrenia for school-age children, present episode version. Arch Gen Psychiatry 1985; 42: 696-702.

5 Cohen J. A coefficient of agreement for nominal scales. Educ Psychol Measurement 1960; 20: 37-46.

6 Endicott J, Spitzer R, Fleiss J, Cohen J. The Global Assessment Scale: a procedure for measuring overall severity of psychiatric disturbance. Arch Gen Psychiatry 1976; 33: 766-71.

7 Endicott J, Spitzer RL. A diagnostic interview: the schedule for affective disorders and schizophrenia. Arch Gen Psychiatry 1978; 35: 837-44.

8 Kaufman J, Birmaher B, Brent D, Rao U, Flynn C, Moreci $\mathrm{P}$, et al. Schedule for affective disorders and schizophrenia for school-age children-present and lifetime version (K-SADS-PL): initial reliability and validity data. J Am Acad Child Adolesc Psychiatry 1997; 36(7): 980-8

9 Madianos MG. The reliability and validity of the Global Assessment Scale in the Greek population. Encephalos 1987; 24: 97-100 [in Greek].

10 Shaffer D, Gould M, Brasic J, Ambrosini P, Fisher P, Bird H, et al. A Children's Global Assessment Scale (CGAS). Arch Gen Psychiatry 1983; 40: 1228-31. 